\title{
SOLUBILIZATION OF SOME NON-STEROIDAL ANTI- INFLAMMATORY DRUGS (NSAIDs) BY PLURONIC F-127 BLOCK COPOLYMER
}

\author{
M. EL-Badry, M. Fathy and M. G. Abdel Mohsen
}

Department of Pharmaceutics, Faculty of Pharmacy, Assiut University, Assiut, Egypt

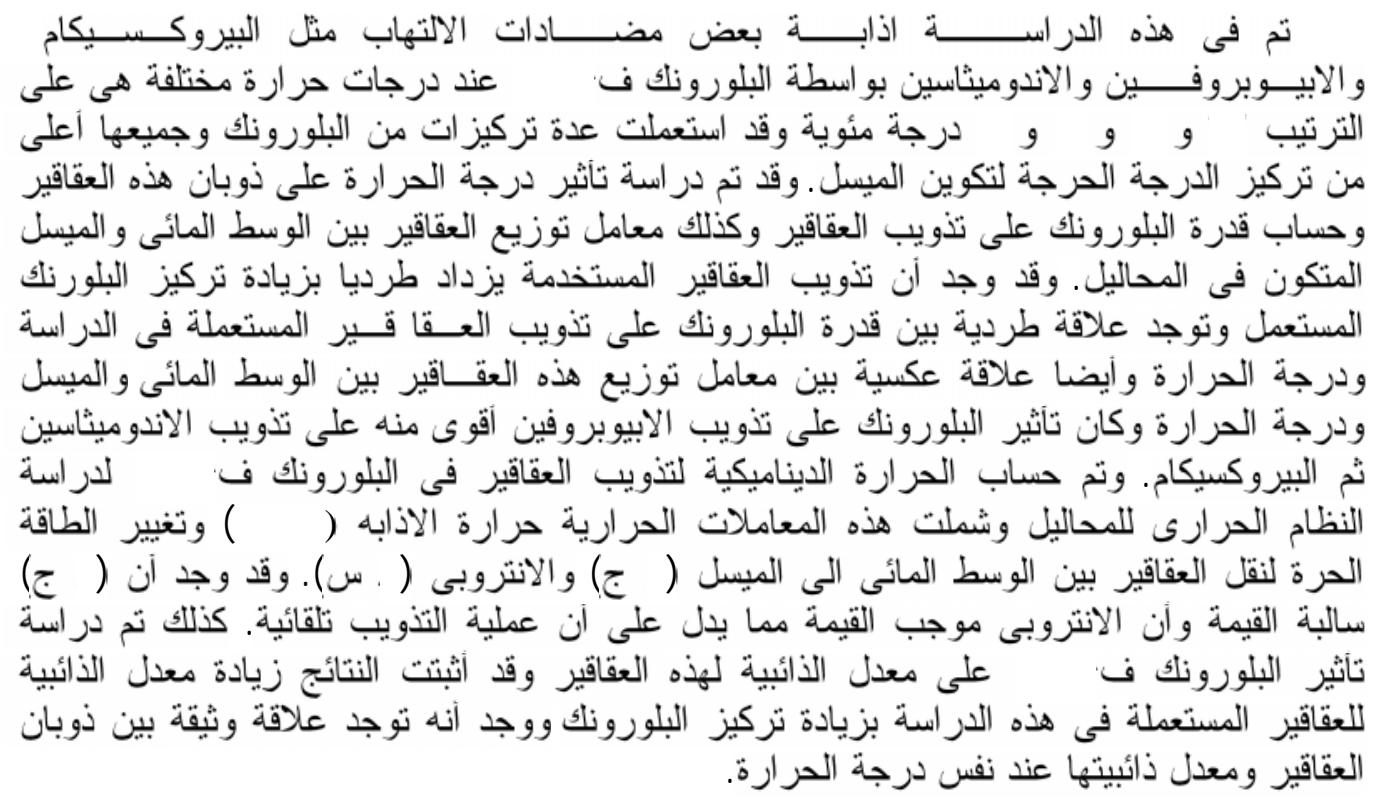

This work is an attempt to solubilize certain non-steroidal anti-inflammatory drugs such as Piroxicam (PXM), Ibuprofen (IBU) and Indomethacin (IND), through the use of hydrophilic surfactant pluronic F-127 (Pl. F-127), which was used in different concentrations above its critical micelle concentration (CMC). The solubility of the investigated drugs was enhanced, and was linearly correlated with the surfactant concentration. The effect of temperature on the solubilization capacity of the surfactant and the distribution coefficient of the drugs between the micellar and aqueous phases was determined. It was found that, there is a direct relationship between the solubilization capacity of surfactant toward the used drugs and temperature and there is an inverse relationship between the distribution coefficient and temperature. These observations were more obvious for IBU than IND and PXM.

The change in heat of solution $(\Delta H)$, free energy $(\Delta G)$ and entropy $(\Delta S)$ as a result of solubilization of the investigated drugs were calculated. The data prevailed that, the presence of Pl. F-127 energetically favors the solubility of the used drugs and more favorable by increasing the surfactant concentration. The dissolution study was performed for these drugs. Pl. F-127 proved its ability to enhance the dissolution rate of the investigated water insoluble drugs.

\section{INTRODUCTION}

Solubilization refers to the phenomenon of an increase in the solubility of insoluble substances in a solvent medium caused by the presence of amphiphilar aggregates. The aggregates provide a microenvironment conducive to the solubilizate molecules, which are otherwise only sparingly soluble in the bulk solvents. In aqueous solutions containing 
surfactant micelles, the solubility of hydrophobic solutes is increased by many orders of magnitude. ${ }^{1}$

Solubilization in surfactant solutions above the critical micelle concentration offers a good approach to the formulation of water insoluble drugs. This technique has attracted great attention, not only for its intrinsic scientific interest but also it has many technical applications. $^{2-5}$

The drugs, which were used in this study are Piroxicam (PXM), Ibuprofen (IBU) and Indomethacin (IND), they are non-steroidal anti-inflammatory drugs (NSAIDs) and also possesses analgesic properties. They are poorly water-soluble drugs and therefore their dissolution would be limited. By increasing their water solubility, their bioavailability will be improved. ${ }^{6-8}$

Pluronic F-127 (Pl.F-127) is one of many polyoxyethylene- polyoxypropylenepolyoxyethylene type block copolymers, It is a nonionic surfactant and it has many useful characteristics such as low toxicity and gelling property. ${ }^{9}$ Also it has been employed as a vehicle in various pharmaceutical dosage forms. ${ }^{10-13}$

In the present work different non-steroidal anti-inflammatory drugs namely Piroxicam (PXM), Ibuprofen (IBU) and Indomethacin (IND) were solubilized by different concentrations of pluronic F-127 above its critical micelle concentration (CMC) and at different temperatures, then the solubilizing capacity of the surfactant was calculated, Van't Hoff plots were performed for the drugs in the investigated solutions. Furthermore, the thermodynamic parameters of the solubilizied drugs were determined. Also the dissolution rates of drugs in different concentrations of pluronic solutions were determined.

\section{EXPERIMENTAL}

\section{Materials}

Piroxicam (Sedico Chemical Company Cairo, Egypt), Indomethacin, Ibuprofen (Egyptian International Pharmaceutical Co., Egypt), Pluronic F-127 (BASF Aktiengeseilschaft, Ludwigshafen, Germany). Other materials and solvents are of reagent or analytical grades.

\section{Methods}

Solubility measurements

Excess quantities of different drugs were placed in glass-stoppered bottles together with $20 \mathrm{ml}$ of aqueous solutions of different concentrations of pluronic F-127 (0.03, 0.06, 0.125 and $0.25 \mathrm{mM}$ ). Solutions were shaken for $24 \mathrm{hr}$. into a shaking water bath (SBS Instruments, Germany) at different temperatures $\left(37,45,50\right.$ and $\left.60^{\circ}\right)$ and controlled within $\pm 0.5^{\circ}$. This period was found to be sufficient for attaining equilibrium. After the equilibrium was established, samples were filtered through $0.45 \mu \mathrm{m}$ Millipore membrane filter and assayed spectrophotometrically (UV. 1601- Shimadzu Co., Japan) at $334 \mathrm{~nm}$ for piroxicam, ${ }^{14}$ at $265 \mathrm{~nm}$ for Ibuprofen ${ }^{15}$ and at $318 \mathrm{~nm}$ for Indomethacin ${ }^{16}$ against a suitable blank containing the corresponding surfactant solution.

\section{Dissolution rate studies}

The dissolution rate of the drugs was studied using USP dissolution apparatus (Erwicka Co. Germany). Accurately weighed piroxicam, indomethacin and ibuprofen (10, 50 and $200 \mathrm{mg}$ respectively) were dispersed in the dissolution medium $(900 \mathrm{ml}$ of surfactant solutions) at $37 \pm 0.5^{\circ}$ which was immediately stirred at $50 \mathrm{rpm}$. At different appropriate time intervals, $5.0 \mathrm{ml}$ samples were withdrawn, filtered (if necessary), (diluted if necessary, for low concentration of pluronic the dilution was $1: 5$ and for high concentration it was 1:10) and the absorbance was measured. Equal volumes of fresh dissolution medium was replaced to keep the volume constant. The cumulative amounts of the drugs dissolved were calculated.

\section{RESULTS AND DISCUSSION}

For the development of a liquid pharmaceutical formulations, the solubility of the active ingredients in the vehicle is often an important factor to determine the suitable dose. The poor solubility of anti-inflammatory drugs limiting their use in aqueous preparations. Pluronic F-127, a hydrophilic surfactant, is used to enhance the solubility of these drugs. 
Effect of different concentrations of Pl. F127 on the aqueous solubility of the used drugs

The solubilization of PXM, IBU, and IND in different concentrations of aqueous solutions of Pl. F-127 (0.03, 0.06, 0.125 and $0.25 \mathrm{mM})$ at different temperatures were shown in Figures 1-3. It is obvious that the solubility of these drugs were increased in the presence of the surfactant. For the highest concentration of $\mathrm{Pl}$. F-127 $(0.25 \mathrm{mM})$ and at $37^{\circ}$, approximately, seven-fold, fifty-fold and two-fold increase was noted for IBU, IDN, and PXM respectively. The solubilization of drugs in a surfactant solution has been suggested to occur due to the increased partitioning of solute molecules into the micelles, which could be considered as a separate, pseudo phase. ${ }^{17}$ The value of the critical micelle concentration (CMC) for Pl. F127 is $0.01 \mathrm{mM}^{18}$ which was small compared to the concentrations used in this study (0.03-0.25 $\mathrm{mM})$. The solubilizing effect of Pl. F-127 was more pronounced on the IND followed by IBU and lastly PXM. This result is a reflection of the lowest solubility of IND in pure water. ${ }^{8}$ The higher solubilizing effect of Pl.F-127 on IBU than PXM might due to the smaller molecular of IBU, which help the molecule to be easily inserted into the micelle.

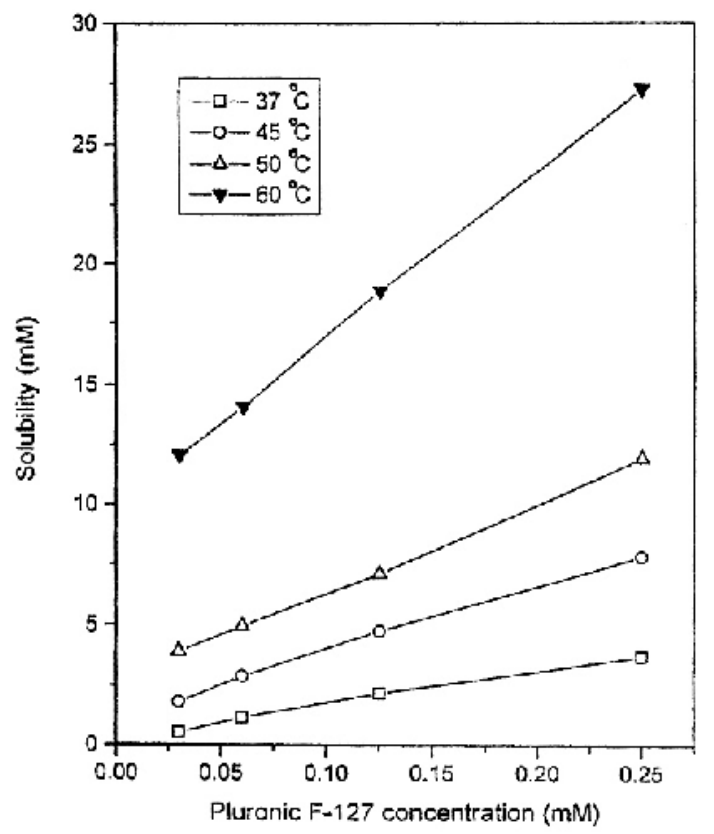

Fig. 1: Solubility profiles of Ibuprofen in different concentrations of aqueous solutions of pluronic F-127 at different temperatures.

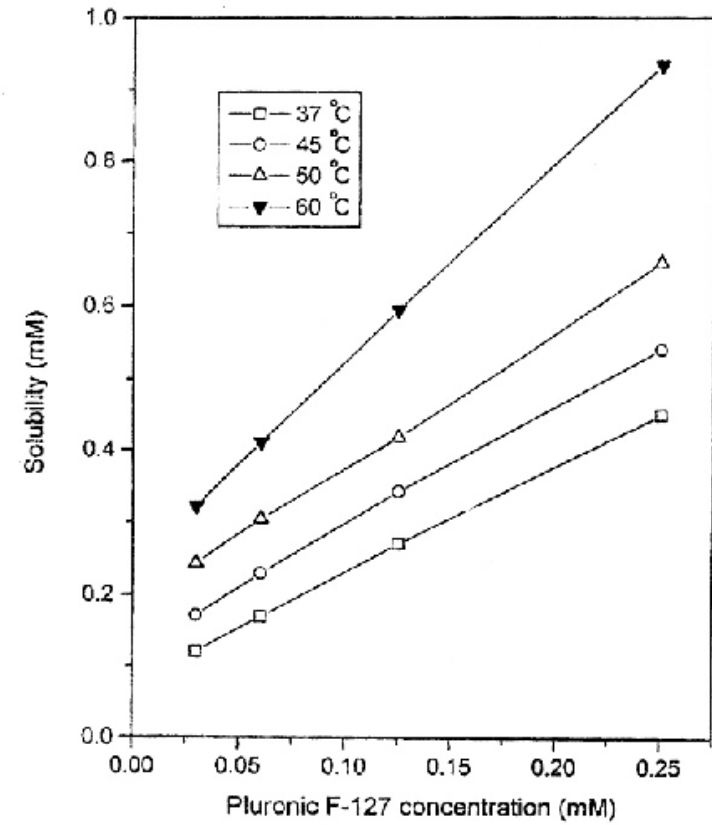

Fig. 2: Solubility profiles of Indomethacin in different concentrations of aqueous solutions of pluronic F-127 at different temperatures.

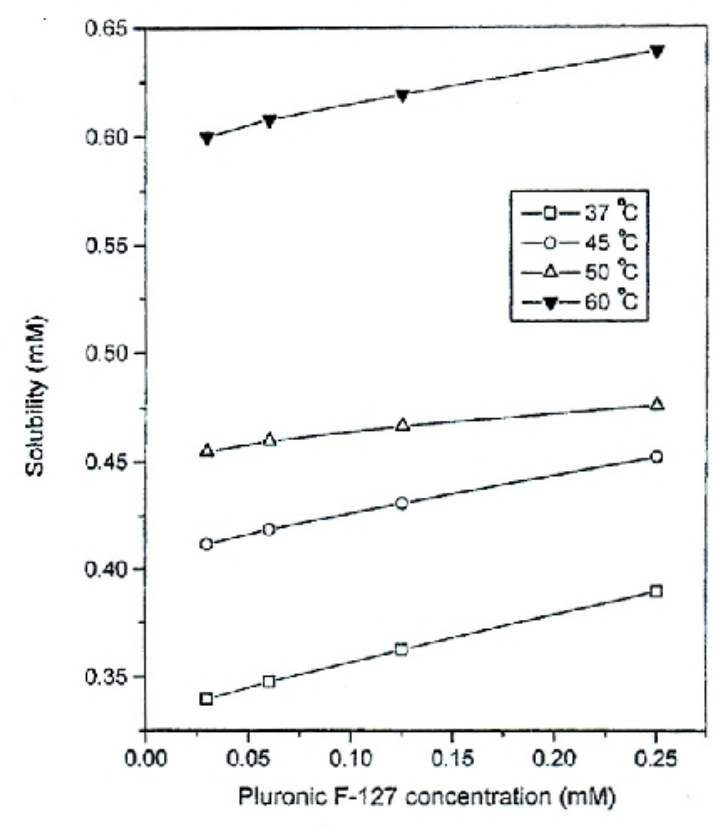

Fig. 3: Solubility profiles of Piroxicam in different concentrations of aqueous solutions of pluronic F-127 at different temperatures. 
In general, the plots of drug solubility against surfactant concentrations at the investigated temperatures indicated a linear relationship in the predetermined concentration range $(0.03-0.25 \mathrm{mM})$. These results were in agreement with that reported by Suh and Jun, ${ }^{9}$ they reported that, the linear increase in the solubility of naproxine as a function of Pl.F127 concentration and they suggested that the total micellar volume into which the drug partitioned was depend on the concentration of Pl. F-127 present.

The solubilization of a slightly watersoluble compound can be treated in terms of association equilibrium between the solutes and the micelles in a micellar solution. ${ }^{19}$ Thus:

$\mathrm{D}_{\mathrm{w}}+\mathrm{M} \stackrel{\mathrm{k}_{\mathrm{m}}}{\leftrightarrows} \mathrm{D}_{\mathrm{m}}$

Where $D_{m}$ and $D_{w}$ are the drug solubilized in the micellar and bulk water, $\mathrm{M}$ is the micellar concentration and $\mathrm{k}_{\mathrm{m}}$ is the distribution coefficient of the drug between the micellar and bulk aqueous phases.

Accordingly, the following relationships were derived:

$\mathrm{K}_{\mathrm{m}}=\mathrm{S}_{\mathrm{m}} / \mathrm{S}_{\mathrm{w}} \mathrm{M}$

and $\mathrm{S}_{\mathrm{t}}=\mathrm{S}_{\mathrm{m}}+\mathrm{S}_{\mathrm{w}}$

so $S_{\mathrm{t}}=\mathrm{k}_{\mathrm{m}} \mathrm{S}_{\mathrm{w}} \mathrm{M}+\mathrm{S}_{\mathrm{w}}$
Where $S_{\mathrm{m}}$ and $S_{\mathrm{w}}$ are the solubility of the drug in the micellar and bulk aqueous phases, respectively, $\mathrm{M}$ is the molar concentration of the surfactant solution and $\mathrm{S}_{\mathrm{t}}$ is the total molar solubility of the drug in the micellar solution. Therefore, a plot of $S_{t}$ versus $M$ will produce a straight line with an intercept of $S_{w}$ and a slope of a $K_{m} S_{w}$, which is the solubilizing capacity of the micelles. The slopes were determined by linear regression and the distribution coefficient $\left(\mathrm{k}_{\mathrm{m}}\right)$ can be calculated.

Table 1 presents the solubilizing capacity and distribution coefficient of IBU, IND and PXM between the micellar and bulk aqueous phases. It was found that the solublizing capacity of Pl. F-127 towards the used drugs could be ranked in this manner Ibuprofen > Indomethacin > Piroxicam. This is due to the difference in the molecular weights of drugs, where IBU has the smallest while, there is a small difference between IND and PXM in the molecular weight. It was also found that, the solubilizing capacity of Pl. F-127 towards IBU and IND increases significantly by increasing temperature while a slight increase was observed in case of PXM. The distribution coefficient $\left(\mathrm{k}_{\mathrm{m}}\right)$ of IBU and IND decreased by increase the temperature but still constant in case of PXM.

Table 1: Effect of different temperatures on the solubilizing capacity and distribution coefficient of Ibuprofen, Indomethacin, Piroxicam between micellar solution of pluronic F-127 and aqueous phases.

\begin{tabular}{|c|c|c|c|c|c|c|}
\hline \multirow{2}{*}{ Temperature } & \multicolumn{2}{|c|}{ Ibuprofen } & \multicolumn{2}{c|}{ Indomethacine } & \multicolumn{2}{c|}{ Piroxicam } \\
\cline { 2 - 7 } & $\begin{array}{c}\text { Solubilizing } \\
\text { Capacity }\end{array}$ & $\begin{array}{c}\text { Distribution } \\
\text { Coefficient } \\
\left(\mathrm{mM}^{-1}\right)\end{array}$ & $\begin{array}{c}\text { Solubilizing } \\
\text { capacity }\end{array}$ & $\begin{array}{c}\text { Distribution } \\
\text { Coefficient } \\
\left(\mathrm{mM}^{-1}\right)\end{array}$ & $\begin{array}{c}\text { Solubilizing } \\
\text { capacity }\end{array}$ & $\begin{array}{c}\text { Distribution } \\
\text { Coefficient } \\
\left(\mathrm{mM}^{-1}\right)\end{array}$ \\
\hline $37^{\circ}$ & 13.60 & 37.73 & 1.225 & 11.97 & 0.1900 & 0.200 \\
\hline $45^{\circ}$ & 25.59 & 18.69 & 1.435 & 11.85 & 0.1901 & 0.200 \\
\hline $50^{\circ}$ & 35.44 & 11.87 & 1.945 & 11.11 & 0.1909 & 0.204 \\
\hline $60^{\circ}$ & 72.67 & 7.55 & 2.58 & 11.05 & 01990 & 0.240 \\
\hline
\end{tabular}

The solubilizing capacity $\left(\mathrm{K}_{\mathrm{m}} \mathrm{S}_{\mathrm{w}}\right)$ and distribution coefficient $\left(\mathrm{k}_{\mathrm{m}}\right)$, were calculated by plot of $\mathrm{S}_{\mathrm{t}}$ (solubility of drug) versus $\mathrm{M}$ (molar concentration of surfactant will produce a straight line with an intercept of $S_{w}$ and a slope of a $K_{m} S_{w}$, which is the solubilizing capacity of the micelles. The slopes were determined by linear regression and the distribution coefficient $\left(\mathrm{k}_{\mathrm{m}}\right)$ can be calculated. 
These results were in agreement with Suh and Jun, ${ }^{9}$ the authors reported that, the size of micelles was increased by increasing the temperature until a threshold temperature, approximately $20^{\circ}$ below the cloud point of the surfactants, was reached. They showed that, the micellar volume expands rapidly and asymmetrically, thus causing an increase in drug loading into the micelles. In this experiment, the highest temperature used was $60^{\circ}$, far below the cloud point of Pl. F-127, which is to be $100^{\circ}$.

\section{Thermodynamic Parameters}

Thermodynamic parameter enthalpy change $(\Delta \mathrm{H})$, free energy change $(\Delta \mathrm{G})$, entropy change $(\Delta S)$ associated with the solubilization of the tested drugs in the Pl. F-127 solution was calculated. These parameters are particularly useful in understanding the thermodynamic phenomenon involved in the micellar solubilization.

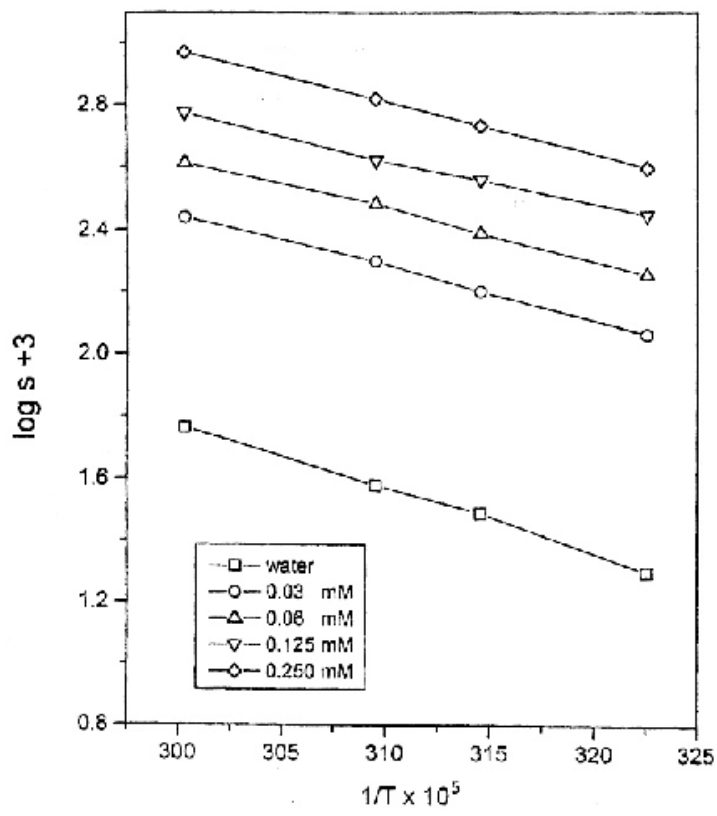

Fig. 4: Van't Hoff plot of Ibuprofen in aqueous solutions of pluronic F-127.

It was evident from Figures 4-6 that, the solubility increased in all cases with increasing temperature indicating that the solution process is endothermic, thus heat is absorbed when drug was dissolved. The values of the heat of solution $(\Delta \mathrm{H})$ (Tables 2-4) were calculated from the slopes of vant' Hoff plot using the Sokoloski formula. ${ }^{20}$ $\ln \mathrm{C}_{\mathrm{s}}=\Delta \mathrm{H} / \mathrm{RT}+\mathrm{J}$

Where $\mathrm{C}_{\mathrm{s}}$ is the solubility, $\Delta \mathrm{H}$ is the heat of solution (cal/mole), $\mathrm{R}$ is the molar gas constant (1.987 cal./deg. mole), $\mathrm{T}$ is the absolute temperature $(\mathrm{K})$ and $\mathrm{J}$ is a constant.

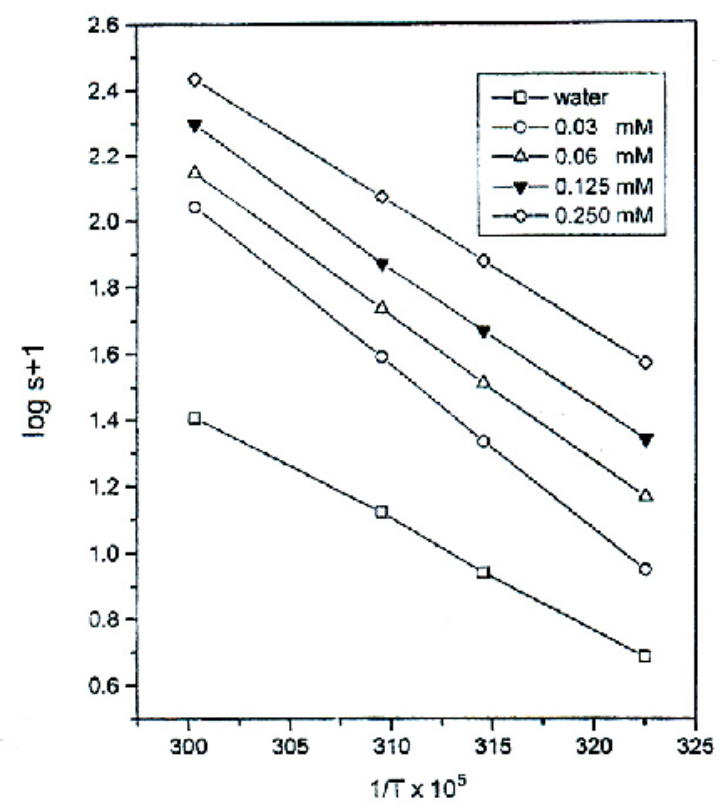

Fig. 5: Van't Hoff plot of Indomethacine in aqueous solutions of pluronic F-127.

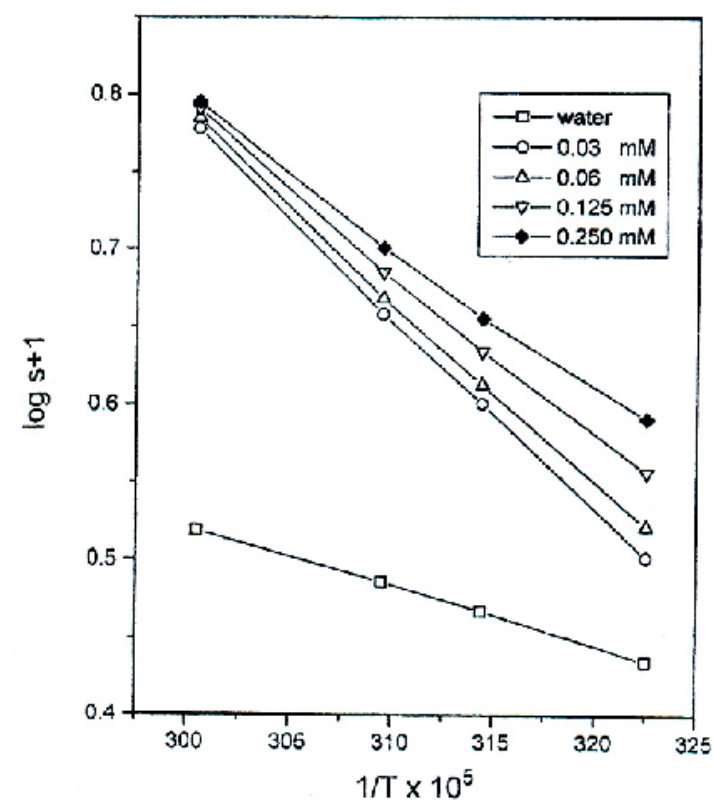

Fig. 6: Van't Hoff plot of Piroxicam in aqueous solutions of pluronic F-127. 
Table 2: Thermodynamic parameters for the solubilization of Ibuprofen in different concentrations of pluronic F-127 solution.

\begin{tabular}{|c|c|c|c|c|c|c|c|c|c|}
\hline \multirow{3}{*}{$\begin{array}{c}\text { Pluronic } \\
\text { F-127 conc. } \\
(\mathrm{mM})\end{array}$} & \multirow{3}{*}{$\mathrm{H}$} & \multicolumn{8}{|c|}{ Temperature } \\
\hline & & \multicolumn{2}{|c|}{$37^{\circ}$} & \multicolumn{2}{|c|}{$45^{\circ}$} & \multicolumn{2}{|c|}{$50^{\circ}$} & \multicolumn{2}{|c|}{$60^{\circ}$} \\
\hline & & $\mathrm{G}$ & $\mathrm{S}$ & $\mathrm{G}$ & $S$ & $\mathrm{G}$ & $S$ & $\mathrm{G}$ & $S$ \\
\hline 0.0 & 20.66 & $===$ & $===$ & $===$ & $===$ & $===$ & $===$ & $===$ & $===$ \\
\hline 0.03 & 20.06 & -0.667 & 649 & -0.4421 & 644 & -0.6941 & 642 & -0.3600 & 613 \\
\hline 0.06 & 20.01 & -0.678 & 667 & -0.8448 & 655 & -0.9089 & 647 & -0.5202 & 616 \\
\hline 0.125 & 19.62 & -0.924 & 662 & -1.0850 & 651 & -1.0806 & 641 & -0.7480 & 612 \\
\hline 0.250 & 17.80 & -1.252 & 615 & -1.3835 & 603 & -1.4101 & 595 & -0.9573 & 563 \\
\hline
\end{tabular}

$\mathrm{H}$ : the enthalpy change ( $\mathrm{k}$ cal./mole)

$\mathrm{G}$ : the free energy change ( $\mathrm{k}$ cal./mole)

$\mathrm{S}$ : the entropy change (cal./deg. mole)

Table 3: Thermodynamic parameters for the solubilization of Indomethacin in different concentrations of pluronic F-127 solution.

\begin{tabular}{|c|c|c|c|c|c|c|c|c|c|}
\hline \multirow{3}{*}{$\begin{array}{c}\text { Pluronic } \\
\text { F-127 } \\
\text { conc. }(\mathrm{mM})\end{array}$} & \multirow{3}{*}{$\mathrm{H}$} & \multicolumn{8}{|c|}{ Temperature } \\
\hline & & \multicolumn{2}{|c|}{$37^{\circ}$} & \multicolumn{2}{|c|}{$45^{\circ}$} & \multicolumn{2}{|c|}{$50^{\circ}$} & \multicolumn{2}{|c|}{$60^{\circ}$} \\
\hline & & $\mathrm{G}$ & $\mathrm{S}$ & G & $\mathrm{S}$ & G & $\mathrm{S}$ & G & $\mathrm{S}$ \\
\hline 0.0 & 18.13 & $===$ & $===$ & $===$ & $===$ & $===$ & $===$ & $===$ & $===$ \\
\hline 0.03 & 8.128 & -1.535 & 311 & -1.408 & 299 & -1.138 & 286 & -1.080 & 276 \\
\hline 0.06 & 7.436 & -1.779 & 297 & -1.634 & 285 & -1.336 & 2715 & -1.295 & 262 \\
\hline 0.125 & 6.672 & -2.054 & 281 & -1.881 & 269 & -1.539 & 254 & -1.540 & 246 \\
\hline 0.250 & 6.655 & -2.267 & 287 & -2.134 & 276 & -1.833 & 262 & -1.840 & 255 \\
\hline
\end{tabular}

$\mathrm{H}$ : the enthalpy change ( $\mathrm{k}$ cal./mole)

$\mathrm{G}$ : the free energy change ( $\mathrm{k}$ cal./mole)

$\mathrm{S}$ : the entropy change (cal./deg. mole)

Table 4: Thermodynamic parameters for the solubilization of Piroxicam in different concentrations of pluronic F-127 solution.

\begin{tabular}{|c|c|c|c|c|c|c|c|c|c||}
\hline Pluronic & \multirow{2}{*}{$\begin{array}{c}\text { F-127 } \\
\text { conc. }(\mathrm{mM})\end{array}$} & \multirow{2}{*}{$\mathrm{H}$} & \multicolumn{9}{|c||}{$37^{\mathbf{0}}$} & \multicolumn{2}{|c|}{$45^{\circ}$} & \multicolumn{2}{|c||}{$50^{\circ}$} & \multicolumn{2}{c||}{$60^{\circ}$} \\
\cline { 3 - 10 } & & $\mathrm{G}$ & $\mathrm{S}$ & $\mathrm{G}$ & $\mathrm{S}$ & $\mathrm{G}$ & $\mathrm{S}$ & $\mathrm{G}$ & $\mathrm{S}$ \\
\hline 0.0 & 4.99 & $===$ & $===$ & $===$ & $===$ & $===$ & $===$ & $===$ & $===$ \\
\hline 0.03 & 5.02 & -0.135 & 166 & -0.2139 & 165 & -0.2391 & 163 & -0.410 & 163 \\
\hline 0.06 & 4.90 & -0.149 & 165 & -0.2256 & 163 & -0.2465 & 161 & -0.4205 & 161 \\
\hline 0.125 & 4.77 & -0.172 & 159 & -0.2423 & 158 & -0.2558 & 157 & -0.4315 & 156 \\
\hline 0.250 & 4.22 & -0.219 & 143 & -0.2728 & 141 & -0.2685 & 139 & -0.4464 & 140 \\
\hline
\end{tabular}

$\mathrm{H}$ : the enthalpy change (k cal./mole)

$\mathrm{G}$ : the free energy change ( $\mathrm{k}$ cal./mole)

$\mathrm{S}$ : the entropy change (cal./deg. mole) 
The free energy change $(\Delta \mathrm{G})$ for the transfer of the drug from the aqueous phase to that in water to micellar phase was calculated from the following thermodynamic relationship, Feldman and Gibaldi: ${ }^{21}$

$\Delta \mathrm{G}=-2.303 \mathrm{RT} \log \mathrm{S}_{\mathrm{s}} / \mathrm{S}_{\mathrm{w}}$

Where $S_{w}$ and $S_{s}$ are the solubilities of the drug in water and solubilizing agent, respectively, and $\mathrm{T}$ is the absolute temperature.

Free energy $(\Delta \mathrm{G})$ is defined as $\Delta \mathrm{G}=\Delta \mathrm{H}$ $\mathrm{T} \Delta \mathrm{S}$, where $\Delta \mathrm{S}$ is entropy. So, the entropy of solubilization could be calculated.

The values of $\Delta \mathrm{H}, \Delta \mathrm{G}$ and $\Delta \mathrm{S}$ were summarized in Tables 2-4. The values of $\Delta H$ are positive, which indicated that, the micellar solubilization of the drugs is an endothermic process. However, it was noticed that the values of $\Delta \mathrm{H}$ in surfactant solutions are lower than that of water. As the heat required to break up the crystal will be the same for the dissolution in water as in a surfactant, either the heat of solution differs from that in water or an interaction between the drug and the surfactant molecules account for the difference in heat changes. ${ }^{22}$ The decrease in $\Delta \mathrm{H}$ values was found to be in the following descending order IND, IBN and PXM. For all systems (in this study), $\Delta \mathrm{G}$ was a negative value indicating spontaneous solubilization.

At a constant temperature, $-\Delta \mathrm{G}$ decrease with increasing the surfactant concentration; indicating that, the transfer of the drug into the polymer solution is energetically favorable. ${ }^{23}$

The positive values of entropy, $\Delta \mathrm{S}$, suggested a decrease in the degree of ordering of layer of water molecules surrounding the non polar part of pluronic when the drug was dissolved in the micellar phase. During this process, a disruption of the water structure surrounding the hydrophobic drug molecules could increase the entropy of the system. Conversely, the entrapment of the drug molecules within the micelles could restrict its activity, causing a decrease in entropy of the system. However the net decrease in entropy suggested that the disruption of water structure was not sufficient to compensate for the entropy loss due to the micellar entrapment of the drug molecules. ${ }^{23-24}$

\section{Dissolution rate studies}

Figures 7-9 illustrated the dissolution profiles of IBU, IND and PXM in different concentrations of Pl. F-127 solutions. The dissolution rates of the studied drugs increased by increasing the surfactant concentration, this is due to micellar solubilization. The data presented in Table 5 showed that, there was a correlation between the amount dissoluted of the investigated drugs in Pl. F-127 solutions and their solubility in the surfactant at the same temperature $\left(37^{\circ}\right)$. It was found that, there was a very strong correlation between the amount dissoluted and the solubility of IBU $(r=0.977)$, IND $(r=0.911)$ and a strong correlation between the amount dissoluted and the solubility of $\operatorname{PXM}(r=0.897)$.

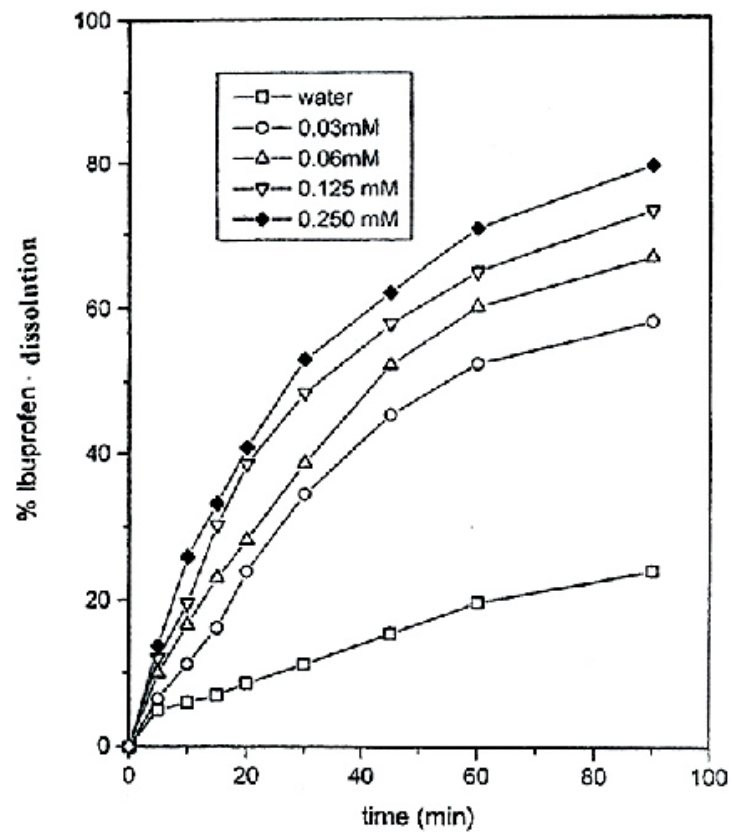

Fig. 7: Dissolution profiles of Ibuprofen in different concentration of aqueous solution of pluronic F-127 at $37^{\circ}$. 


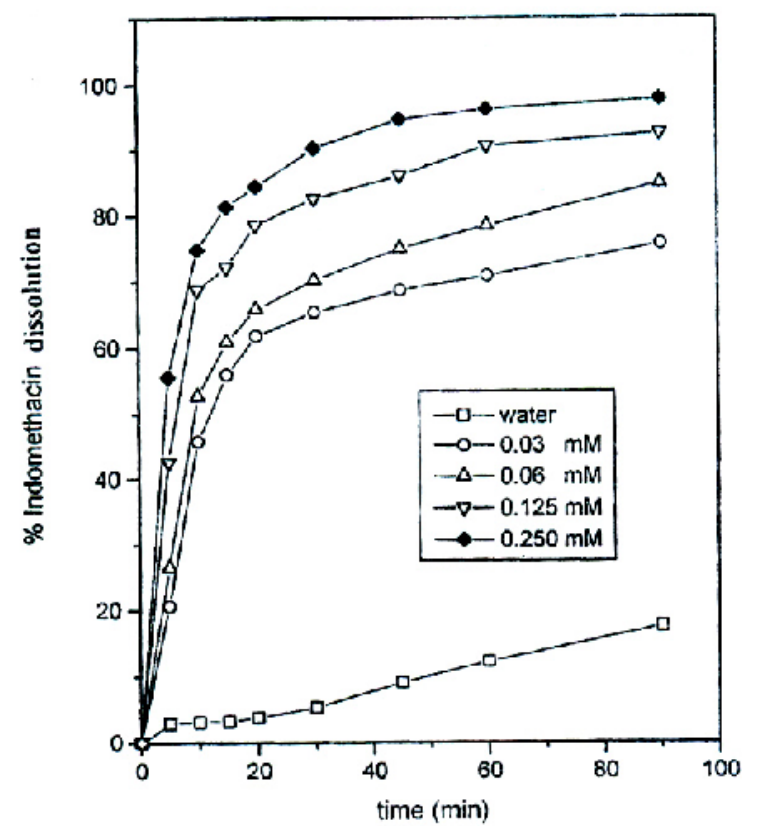

Fig. 8: Dissolution profiles of Indomethacin in different concentration of aqueous solution of pluronic F-127 at $37^{\circ}$.

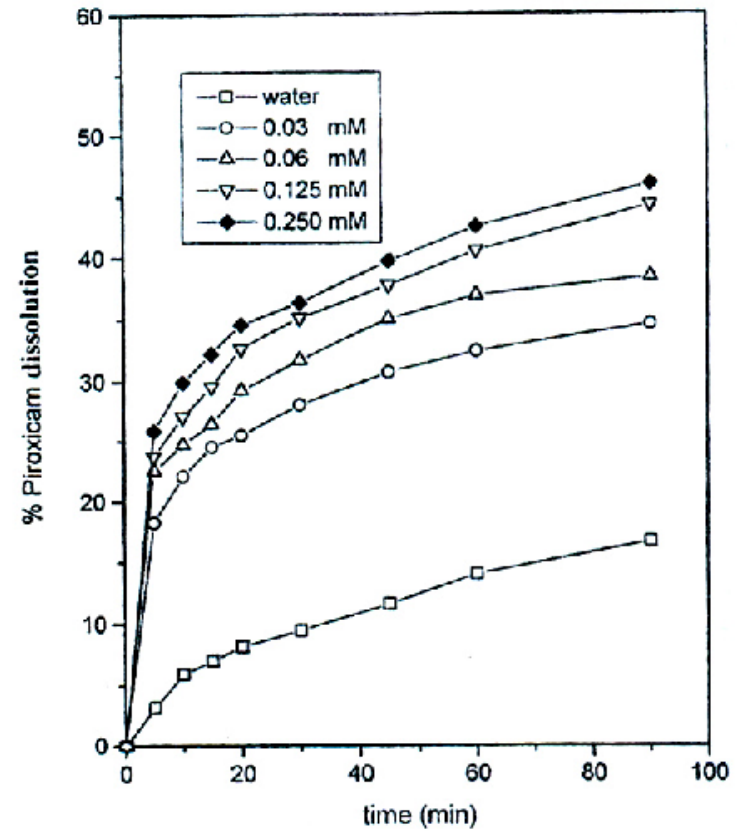

Fig. 9: Dissolution profiles of Piroxicam in different concentration of aqueous solution of pluronic F-127 at $37^{\circ}$.

Table 5: Correlation between the percent amount dissoluted of Ibuprofen, indomethacin and piroxicam and their solubility in pluronic F-127 at $37^{\circ}$

\begin{tabular}{|c|c|c|c|c|c|c|}
\hline \multirow{2}{*}{ Drug } & & \multicolumn{4}{|c|}{ Pluronic F-127 (mM) } & \multirow{2}{*}{$\begin{array}{l}\text { Correlation } \\
\text { coefficient } \\
\text { (r) }\end{array}$} \\
\hline & & 0.03 & 0.06 & 0.125 & 0.25 & \\
\hline \multirow{2}{*}{ Ibuprofen } & $\begin{array}{l}\text { Solubility } \\
\text { (mM) }\end{array}$ & 0.114 & 0.303 & 0.452 & 0.769 & \multirow{2}{*}{0.977} \\
\hline & $\begin{array}{l}\% \text { amount } \\
\text { dissoluted }\end{array}$ & 58.06 & 66.55 & 73.15 & 79.55 & \\
\hline \multirow{2}{*}{ Indomethacin } & Solubility (mM) & 0.010 & 0.180 & 0.281 & 0.397 & \multirow[b]{2}{*}{0.911} \\
\hline & $\begin{array}{l}\% \text { amount } \\
\text { dissoluted }\end{array}$ & 75.80 & 76.92 & 94.5 & 97.76 & \\
\hline \multirow{2}{*}{ Piroxicam } & Solubility (mM) & 0.340 & 0.348 & 0.361 & 0.390 & \multirow[b]{2}{*}{0.897} \\
\hline & $\begin{array}{l}\% \text { amount } \\
\text { dissoluted }\end{array}$ & 34.57 & 38.40 & 44.30 & 45.96 & \\
\hline
\end{tabular}




\section{Conclusion}

Solubility studies showed a solubilizing effect of Pl. F-127 on IBU, IND and PXM. Increasing the temperature enhances the solubilizing capacity of Pl. F-127 toward the used drugs. The negative values of free energy and heat of solution indicated that the presence of Pl. F-127 favors the dissolution of these drugs. Moreover, increasing polymer concentration leads to a further decrease in $\Delta \mathrm{H}$ indicating that the process becomes more favorable with higher polymer concentrations. Despite the close similarity in the values of the heat of solution $(\Delta \mathrm{H})$ for Piroxicam. The free energy changes $(\Delta G)$ at different temperatures indicated that, the spontaneous partitioning of PXM into the micelles of Pl. F-127. Also, Pl. F-127 successfully improved the dissolution rate of the used drugs.

\section{REFERENCES}

1- R. Nagarajan, Colloid and interface Science, 2, 282 (1997).

2- P. Bustamante, M. A. Pena and J. Barra, Int. J. Pharm., 174, 141 (1988).

3- P. Bustamante, A. Martin and M. A. Ganzalez-Guisandoz, J. Pharm. Sci., 82, 635 (1993).

4- H. Scotte, J. Pharm. Sci., 84, 1215 (1995).

5- S. Romero, A. Reillo, B. Escaero and P.Bustamante, Chem. Pharm. Bull. 44 (5) 1061 (1998).

6- T. EL-Faham, M. Mahmoud, and M. A. Hassan, Bull. Pharm. Sci., Assiut University, 23 (2), 91 (2000).

7- G. M. Khan, and Z. Jiabi, Drug Dev. Ind. Pharm., 24 (5), 455 (1998).

8- C. D. Herzfeldt, and R. Kummel, Drug Dev. Ind. Pharm., 9(5) 767 (1983).

9- H. Suh and H. W. Jun, Int. J. Pharm., 129, 13 (1996).

10- C. M. Susan and M. D. Donovan., Int. J. Pharm.12, 147 (1982).
11- S. Miyazaki, S. Takeuchi, C. Yokouchi and M. Takada, Chem. Pharm. Bull., 32 (10) 4205 (1984).

12- S. Miyazakia, T. Nakamura, C. Yoouchi and M. Takada, M., Chem. Pharm. Bull., 35 (3) 1243 (1987).

13- S. H. Khidr, Bull. Pharm. Sci., Assiut uinversity 17 (1) 81 (1994).

14- M. Mihalic, In Flory, K. (Ed.), Analytical Profiles of Drug Substances, Vol (15). Academic Press, London, (1986), pp. 509531.

15- The United State Pharmacopoeia (25), Asian Edition, Welcome Limited, Toronto, Canada, 2002, p. 884.

16- European Pharmacopoeia, (4 ${ }^{\text {th }}$ Ed.), (EDQM) Council of Europe, StrasbourgFrance, 2002, p. 1367.

17- M. E. L. McBain and E. Hutchinson, Solubilization and Related Phenomena, Academic Press, New York (1955).

18- A. Meyer, Ph D. Thesis, Freiburg University, 1985, p. 95.

19- J. T. H. Ong, and E. Manoukian, Pharm. Re., 5, 704 (1988).

20 T.D. Sokoloski, Solutions and Phase Equillibria. In: Osol, A. (Eds) Remington's Pharmaceutical Sciences Mack Publ. Co., Easton, Pennsylvania, Chapter 19, 1970, p 248.

21- S. Feldman, and M.Gibaldi, J. Pharm. Sci., 56, 370 (1976).

22- B. A. Mulley, A. E. Aboutaleb, A. A., Abdel Rahman and S. M. Ahmed, Bull. Pharm. Sci., Assiut Uinversity, 11 (1), (1988).

23- G. R. Lloyd, D. Q. M Craig and A. A Smith, Eur. J. Pharm. and Biopharm., 48, 59 (1999).

24- J. Molpeceres, M. Guzman, P. Bustamante and M. D. R. Aberturas, Int. J. Pharm. 130, 75 (1996). 